\title{
Motion constancy dependent upon perceived distance and the spatial frequency of the stimulus pattern
}

\author{
EUGENE R. WIST, H. C. DIENER, and J. DICHGANS \\ Neurologische Universitätsklinik mit Abteilung für Neurophysiologie \\ Freiburg im Breisgau, West Germany
}

\begin{abstract}
Using a magnitude-estimation technique, the relationship between perceived distance and perceived speed for object-motion perception was determined. It was found that perceived speed increases linearly with perceived distance when angular speed is held constant. Furthermore, it was found that the spatial frequency of a moving periodic stripe pattern potentiates the effect of perceived distance on perceived speed. The slope of the function relating perceived speed and perceived distance was found to increase linearly with increasing spatial frequency. The functional significance of these findings for motion constancy is discussed.
\end{abstract}

Although the existence of motion constancy in object-referred motion perception is indisputable, its underlying basis has been attributed to various mechanisms. Brown (1931) found that the perceived speed of a moving stimulus decreased by only $20 \%$ when its physical distance from the observer was increased from 1 to $10 \mathrm{~m}$, even though a $90 \%$ diminution in its angular speed resulted. Wallach (1939) maintained that this finding was interpretable in terms of Brown's transposition principle (Brown, 1931), according to which the apparent speed of a moving stimulus will remain unaltered, if, when its physical dimensions and those of its surround are decreased by a given proportion, its physical speed is decreased by the same proportion. Since this condition is met when viewing distance of a moving object and its surround is increased, Wallach argued that the transposition principle was sufficient to account for motion constancy, and that, therefore, distance information was superfluous.

Rock, Hill, and Fineman (1968) reported a positive correlation between a measure of size constancy and perceived speed, and concluded that, in addition to the transposition principle, a second motion constancy mechanism relating to size constancy existed. Since, according to the size-distance invariance hypothesis (Kilpatrik \& Ittleson, 1953), perceived distance is involved in size constancy, it is possible that distance information plays a role, after all, in motion constancy. On the basis of the data presented in this paper, we propose that perceived distance does

E. R. Wist on leave from the Whitely Psychology Laboratories, Franklin and Marshall College, Lancaster, Pennsylvania. This study was conducted while this author was a "U.S. Senior Scientist Awardee" of the German Alexander von Humboldt Foundation. Additional support was provided by the Deutsche Forschungsgemeinschaft (SFB 70, "Hirnforschung und Sinnesphysiologie"). in fact influence perceived speed. Moreover, the relationship between perceived distance and perceived speed can be expressed in the form of an equation analogous to that expressing the sizedistance invariance relationship:

$$
M^{\prime}=k \omega D^{\prime},
$$

where $\mathbf{M}^{\prime}=$ perceived speed, $\mathbf{k}=$ constant of proportionality, $\omega=$ the angular speed of stimulus motion, and $\mathrm{D}^{\prime}$ = perceived distance. According to our hypothesis, reflected in Equation 1, when an object moves at a constant linear speed in the frontoparallel plane, the decrease in its angular speed occasioned by an increase in the physical distance of its plane of motion from the observer is compensated by a corresponding increase in perceived distance. In this way, a constant linear, rather than a decreasing, angular velocity would be perceived. When perfect motion constancy exists, $k=1$ in Equation 1.1

The first purpose of this study was to demonstrate the existence of a distance-dependent motionconstancy mechanism and to determine its strength as reflected by $\mathbf{k}$ in Equation 1. Perceived distance was varied by means of the Pulfrich stereoeffect (Pulfrich, 1922) in order to hold both $\omega$ in Equation 1 and the spatial parameters of the moving retinal images constant. The second purpose of this study relates to our previously reported finding that for object motion, perceived speed increases linearly as a function of the spatial frequency $\left(f_{s}\right)$ of the moving stimulus pattern according to the equation:

$$
M^{\prime}=\left(k_{1} \cdot f_{s}+k_{2}\right) \omega
$$

(Diener, Wist, Dichgans, \& Brandt, 1976). We suggested, in considering the functional significance 
of this finding, that the spatial frequency effect on perceived speed may play a role in motion constancy via temporal frequency $\left(f_{t}=f_{s} \omega\right)$ in Equation 2 . Consider the case of a vertically oriented periodic pattern moving horizontally in the frontoparallel plane at a given distance from the observer. If the observer's eyes are stationary, the temporal frequency of stimulation of a given region of the retina is equal to the product of the spatial frequency (in cycles/deg) and angular speed (in $\mathrm{deg} / \mathrm{sec}$ ) of this stimulus (Diener et al., 1976). If the distance of this moving pattern is now doubled, its angular speed is halved while its spatial frequency is also doubled. But its temporal frequency remains unchanged. Hence, constant temporal frequency could be used by the visual system, in this case, to signal the constant linear speed of the stimulus. Consequently, spatial frequency (via temporal frequency) and distance information may jointly determine motion constancy. In terms of Equation 1, this implies that the value of $k$ is a function of the spatial frequency of the moving pattern. We determined whether this is, in fact, the case in an experiment in which the effects of both variations in perceived distance and spatial frequency on perceived speed were measured.

\section{METHOD}

\section{Subjects}

Thirteen male subjects were employed, 11 of whom were university students naive as to the purpose of the experiment and paid for their services. The other two were staff members. The subjects' ages ranged between 22 and 42 years (median 25 ).

\section{Apparatus}

The subject sat in an upholstered chair fitted with a head support $160 \mathrm{~cm}$ away from a cylindrically shaped screen. A Tönnies optokinetic stimulator was used to project a pattern of alternate dark and light stripes of equal angular width onto the screen. This apparatus is described in detail elsewhere (Diener et al., 1976). Four spatial frequencies were used: 0.022 cycle $/ \mathrm{deg}$ [spatial period $(\lambda)=45 \mathrm{deg}$ ], $0.044 \mathrm{cycle} / \mathrm{deg}(\lambda=22.5 \mathrm{deg})$, $0.066 \mathrm{cycle} / \mathrm{deg}(\lambda=15 \mathrm{deg})$, and $0.088 \mathrm{cycle} / \mathrm{deg}(\lambda=11.25 \mathrm{deg})$. The luminance of the dark stripes was $2.70 \mathrm{~cd} / \mathrm{m}^{2}$, while that of the light ones was $18.62 \mathrm{~cd} / \mathrm{m}^{2}$. The contrast ratio was .75 . The stimulus field subtended a visual angle of $22 \mathrm{deg}$ in width and $40 \mathrm{deg}$ in height and was centered about a dark fixation point $0.5 \mathrm{deg}$ in diameter located in the median plane at eye level on the surface of the screen.

\section{Procedure}

Variation of the perceived distance of the moving stripe pattern without altering the retinal image characteristics. Variations in perceived distance were produced by taking advantage of the Pulfrich stereophenomenon. As is well known from the work of Pulfrich (1922) and Lit (1949), the magnitude of the change in the perceived depth of the path of motion of a moving stimulus depends upon the luminance difference between the two eyes, the stimulus speed, and viewing distance. In the case of a constantvelocity stimulus moving across the visual field to the left or right, the perceived depth interval between the motion path and a stationary fixation point in the same physical depth plane increases with the density of a neutral filter placed before one eye. When stimulus motion is toward the filter-covered eye, the moving stimulus appears closer than the fixation point. The reverse is true for stimulus motion in the opposite direction. Thus both the perceived relative depth between the moving stimulus and the fixation point and the perceived absolute distance of the moving stimulus from the observer are simultaneously affected by viewing through the filter.

A preliminary experiment was conducted with three additional subjects in order to choose filter-density/stimulus-speed combinations which would yield the desired variation in the perceived distance of the moving stripes in the experiment proper. Since it was desired to produce three different perceived distances of the moving stripes, all of which were in front of the projection screen, the following procedure was followed: A small disk, 0.5 deg in diameter, was suspended by means of a fine thread al a distance of 120,130 , or $145 \mathrm{~cm}$ from the subject $(40,30$, and $15 \mathrm{~cm}$, respectively, in front of the screen) and slightly higher than the fixation point affixed to the screen. Using the method of limits, the angular speed of stripe motion necessary in order to make the stripes appear to be moving at each of the three distances was determined. A $1.5 \mathrm{log}$ unit neutral density filter covered the left eye for stripe motion to the left and the right eye for stripe motion to the right. Each subject received a total of 24 trials ( 3 distances $\times 4$ spatial frequencies $\times 2$ motion directions). Since, as was expected, no difference was found between left and right motion directions, these data were combined in calculating the mean angular speeds for the resulting 12 perceived distance-spatial frequency combinations. Arranged in order from the smallest to the largest distance (i.e., 120, 130 , $145 \mathrm{~cm}$ ), the mean angular speeds for the four spatial frequencies rounded to the nearest $5 \mathrm{deg}$ were: for 0.022 and $0.044 \mathrm{cycles} / \mathrm{deg}$, 80,60 , and $40 \mathrm{deg} / \mathrm{sec}$; for $0.066 \mathrm{cycles} / \mathrm{deg}, 60,45$, and $30 \mathrm{deg} / \mathrm{sec}$; and for 0.088 cycles $/ \mathrm{deg}, 55,40$, and $25 \mathrm{deg} / \mathrm{sec}^{2}$ These angular speeds were subsequently employed to yield the desired variation in perceived distance in the experiment proper.

On the basis of this preliminary experiment, it was now possible to produce three different perceived distances for each of the four spatial frequencies of stripe patterns. If, say, the 0.022 -cycle/deg spatial frequency pattern moving at $80 \mathrm{deg} / \mathrm{sec}$ was observed with the $1.5 \log$ unit filter over one eye, it would appear to be moving in a plane about $120 \mathrm{~cm}$ from the eyes $(40 \mathrm{~cm}$ in front of the projection screen). If this filter was removed while continuing to observe the moving pattern, two changes were observed. One was that, in accordance with the Pulfrich effect, the plane of stripe motion shifted immediately back to the surface of the screen. The other was that the apparent speed of the stripes simultaneously increased even though the angular speed of pattern motion had remained constant. It must be stressed that this increase in perceived speed upon removal of the filter was not due to the resulting increment in pattern luminance, since, with monocular viewing, removal of the filter did not lead to an increase in apparent speed. ${ }^{3}$ If this same stimulus pattern was observed moving at $40 \mathrm{deg} / \mathrm{sec}$ with the $1.5 \mathrm{log}$ unit filter over one eye, it appeared to be moving in a plane about $145 \mathrm{~cm}$ from the eyes $(15 \mathrm{~cm}$ in front of the screen). If the filter was removed, again a shift in the plane of motion to the distance of the screen occurred simultaneously with an increase in apparent speed. This apparent speed increase, however, was smaller than in the first example. Thus a smaller increment in apparent speed was associated with a smaller increment in perceived distance. The magnitude of the apparent speed change, while holding angular speed constant appeared proportional to the magnitude of the apparent distance change, as indicated in Equation 1.

In order to obtain quantitative data on this phenomenon, a modified magnitude-estimation technique (Stevens, 1957) was used for the determination of both perceived speed and distance. A different modulus was employed for each angular speed of stripe motion and for each spatial frequency. This modulus condition and the one whose speed or distance was to be estimated differed in only one respect: the density of the filter covering one of the subject's eyes. The angular speed and spatial frequency of 
the moving pattern was therefore identical for both the modulus condition and the test trial on which an estimate of speed or distance was to be made. The modulus condition for speed estimates was always assigned the arbitrary value of " 20 " and was always observed with the $1.5 \log$ unit neutral density filter over one eye, such that the plane of stripe motion was perceived by the subject as closer than the distance of the projection screen. After viewing the modulus condition for $15 \mathrm{sec}$, the subject was instructed to remove the filter and view the moving stripes with a $0.2 \log$ unit filter in its place. This latter filter was not of sufficient density to prevent the stripes from now appearing to move in the plane of the projection screen. If the stripes appeared to be moving faster with this filter than under the modulus condition with the $1.5 \mathrm{log}$ unit filter, then the subject was to assign a number greater than 20 to their perceived speed, with the size of this number being proportional to the increase in apparent speed of the stripes. If they appeared to be moving more slowly, a proportionally lower number was to be assigned. In the event of no change in apparent speed, the modulus value of 20 was to be assigned.

After making a magnitude estimate of speed on a given trial with a given spatial-frequency/angular-speed combination, a new combination was introduced and viewed with the 1.5 log unit filter over the appropriate eye. This constituted a new modulus condition again with the arbitrary value of " 20 ." Then the filter was replaced by the $0.2 \log$ unit filter which shifted the perceived distance of the moving stripe pattern away from the subject to the plane of the projection screen. At this point, another magnitude estimate of speed was made based on the new modulus condition. This procedure was repeated for all combinations of angular speed and spatial frequency.

For magnitude estimates of perceived distance, the procedure followed was identical to that for perceived speed estimates, except that, for the standard conditions, the moving stripes were viewed through the $0.2 \log$ unit filter so that they appeared to be at the distance of the projection screen. In addition, the arbitrary value of "100" was assigned to this distance as the modulus. After viewing a given standard for $15 \mathrm{sec}$, the subject was instructed to replace the 0.2 with the $1.5 \mathrm{log}$ unit filter and to note whether the apparent distance of the moving stripes had altered. If it had, he was to report this change by assigning a number proportionally smaller or larger than 100 , depending upon whether the stripes had appeared to move closer or farther, respectively, from the screen. As for magnitude estimates of apparent speed, angular speed and spatial frequency were identical for standard and test stimuli within a given trial. Within a given trial, only filter density, and consequently perceived distance, was altered.

Different modulus values for estimates of speed and distance were deliberately chosen in order to hinder comparisons by subjects between these estimates. Moreover, on a given trial, only a single estimate of speed or distance was made and the order of presentation of the various conditions was randomized to further hinder such comparisons. The fact that a given modulus was associated with different standard conditions over trials was not found to be confusing by the subjects, since a given standard stimulus was always presented just prior to the required estimate. Thus the perceived speed or distance associated with a particular modulus had to be retained for only several seconds prior to the making of a magnitude estimation.

The 13 subjects were run in two groups of 10 subjects each. Seven of the subjects served in both groups. Group I received the $11.25-, 15-$, and 30-deg spatial periods, while Group II received the 15 and 45 periods only. Each spatial period of the stripe pattern was presented at each of the three angular speeds determined in the preliminary experiment as producing the desired variation in perceived distance. Each stimulus speed, in turn, was presented twice, once moving to the left and once to the right. Thus, a total of six trials was presented for each spatial period. Each subject received the stimulus conditions in a different random order. All magnitude estimations were made while the subject maintained fixation on the small disk affixed to the surface of the screen. The supplementary marker disk employed in the preliminary experiment was absent. Noise made by the projector motor was masked by music or white noise presented through earphones. A rest period of at least $5 \mathrm{~min}$ was given halfway through the approximately $1-h$ session.

To prevent the possible contaminating influence of motion aftereffects, viewing of each motion stimulus was limited to $15 \mathrm{sec}$, a $1.5-\mathrm{min}$ intertrial interval was used, and the direction of stimulus motion was alternated on successive trials. In pilot studies, motion aftereffects were typically not observed under these conditions, and when they were, they proved to last only a second or two.

Transformation of magnitude estimates of speed. In order to be able to express both speed and distance estimates in equivalent scale units, it was necessary to transform the former estimates. First of all, the modulus for these estimates was " 20 " while that for the distance estimates was "100." Secondly, because the standard conditions for the speed estimates all involved perceived distances of the moving patterns which were nearer than the screen distance (viewing with the $1.5 \log$ unit filter), while those for the distance estimates all involved perceived distances at the surface of the screen $(0.2 \mathrm{log}$ unit filter), a reduction (or increase) in perceived speed and perceived distance was reflected differently in the two cases. Since the standard for distance estimates was at the distance of the screen, an estimate less than " 100 " meant a reduction in perceived distance. But, since the standards for the speed estimates were located at the nearer distances, an estimate greater than 20 meant a reduction in perceived speed at the nearer distance. Thus, if an original estimate were, say, " 25 ," this meant that the stripes were perceived to be moving faster when they appeared to be moving in the plane of the screen than they did at the nearer standard distance. In order to obtain a transformed estimate (TE) which would reflect the decreased perceived speed at the nearer, standard distance in this example, the obtained estimates (OE) were converted as follows: TE $=20 / \mathrm{OE} \times 100$. In the case of the example, the transformed estimate is $20 / 25 \times 100=$ 80. Thus, this transformation simultaneously converts the data to a scale based on 100 , as for the distance estimates, and yields transformed estimates in which lower perceived speeds, like smaller perceived distances, are expressed in terms of a percentage less than 100. For the transformed speed estimates, a perfect invariance between perceived speed and distance would be indicated when $k=1$ in Equation 1, since both speed and distance estimates are commensurate. ${ }^{4}$

\section{RESULTS}

The mean speed and distance estimates are plotted in Figure 1 and are given in the table along with the corresponding standard deviations. This figure and the table show that perceived speed increases with increasing perceived distance, as predicted by Equation 1.

For the sake of clarity, it must be noted again that, while different angular speeds were used to produce the different perceived distances of the moving stripe patterns as indicated in the top three rows of the table, the distance and speed estimates within each row were obtained without altering angular speed. The only difference between the standard stimulus (modulus condition) and the test stimuli within each row was a difference in filter density which left angular speed (and spatial frequency) unchanged. Therefore, to the extent that the estimates deviate from the modulus value of 100 , they indicate changes 


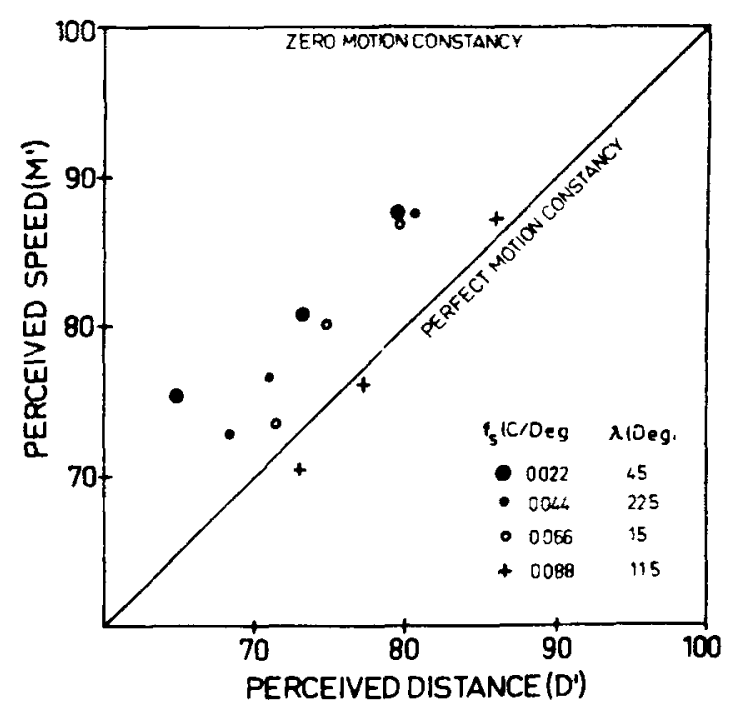

Figure 1. The relationship between perceived distance $\left(D^{\prime}\right)$ and perceived speed $\left(M^{\prime}\right)$ for motion stimuli of constant angular speed differing in spatial frequency $\left(f_{s}\right)$. Each data point represents the mean magnitude estimate of distance and speed for all subjects. (See table for Ns).

in perceived distance in accordance with the Pulfrich effect, and changes in perceived speed positively acorrelated with these perceived distance changes.

A Friedmann analysis of variance indicated a significant difference between the distance estimates plotted on the abscissa $(p<.01)$. This difference simply reflects the success of our manipulation of percejved distance by means of the Pulfrich stereoeffect. More important is the fact that the perceived speed estimates plotted on the ordinate also differed significantly $(p<.01)$, thus indicating a covariation of perceived speed with distance. This covariation is better indicated by the high çorrelation coefficients in the table. Moreover, Figure 1 shows that increas. ing the spatial frequency of the moving stimulus pattern increases the degree of motion constancy. If motion constancy were perfect and independent of $f_{s}$, all points would lie along the diagonal line in Figure 1 labeled "Perfect Motion Constancy," which indicates a perfect invariance between perceived speed and distance. Perceived speed in this case would reflect the linear speed of the stimulus pattern. If zero motion constancy had existed, the data points would be distributed along the upper horizontal line. Perceived speed in this case would reflect the angular speed of the stimulus pattern. This plot shows that at lower spatial frequencies underconstancy exists but at the highest spatial frequency perfect motion constancy is approximated.

These relations were even more clearly reflected in an analysis of the individual data. Regression lines for the functions relating perceived distance and perceived speed for each of the four spatial frequencies were calculated from the data of each subject separately. The means, medians, and standard deviations of the individual slope coefficients are given in the bottom half of the table. The mean slopes and standard deviations are plotted in Figure 2, which shows that the slope, $k$, of Equation 1 increases linearly with increasing spatial frequency." $t$ tests revealed that the mean slopes for the 0.022 - and $0.088-, 0.022$ - and $0.066-$, and $0.044-$ and $0.088-c y c l e / d e g$ spatial frequencies differed significantly $(p<.05)$. Since a slope coefficient of 1.0 is necessary for perfect motion constancy, this figure shows that at the highest spatial frequency tested, $0.088 \mathrm{cycle} / \mathrm{deg}$, a necessary condition for perfect

Table 1

Means and Standard Deviations (SD) of Distance ( $\left.D^{\prime}\right)$ and Speed (MP) Magnitude Estimates (Upper Half)

Measures of Central Tendency, SD, and Correlations Based on Individual Functions (Lower Half)

\begin{tabular}{|c|c|c|c|c|c|c|c|c|c|}
\hline & & & & & atial Pe & n Degree & & & \\
\hline & & & & & & & & & \\
\hline $\begin{array}{l}\text { Angular } \\
\text { Speed }(\omega)\end{array}$ & & $D^{\prime}$ & $\mathbf{M}^{\prime}$ & $D^{\prime}$ & $\mathbf{M}^{\prime}$ & $D^{\prime}$ & $\mathbf{M}^{\prime}$ & $D^{\prime}$ & $\mathbf{M}^{\prime}$ \\
\hline $\begin{array}{l}\text { Highest } \\
\text { Medium } \\
\text { Lowest }\end{array}$ & $\begin{array}{l}\text { Mean } \\
\text { SD } \\
\text { Mean } \\
\text { SD } \\
\text { Mean } \\
\text { SD } \\
\end{array}$ & $\begin{array}{c}66.3 \\
11.56 \\
73.5 \\
7.12 \\
80.7 \\
7.87 \\
\end{array}$ & $\begin{array}{c}75.3 \\
7.33 \\
81.1 \\
7.42 \\
88.1 \\
5.21\end{array}$ & $\begin{array}{c}68.7 \\
11.36 \\
71.8 \\
9.52 \\
80.4 \\
5.75 \\
\end{array}$ & $\begin{array}{l}72.9 \\
11.90 \\
76.6 \\
8.68 \\
87.4 \\
7.58 \\
\end{array}$ & $\begin{array}{c}69.8 \\
9.86 \\
72.2 \\
9.53 \\
78.9 \\
5.25 \\
\end{array}$ & $\begin{array}{c}75.0 \\
9.71 \\
78.1 \\
5.91 \\
86.6 \\
5.92 \\
\end{array}$ & $\begin{array}{c}72.6 \\
9.35 \\
77.8 \\
7.42 \\
85.9 \\
4.18 \\
\end{array}$ & $\begin{array}{l}70.6 \\
12.95 \\
76.0 \\
12.98 \\
86.6 \\
9.91 \\
\end{array}$ \\
\hline $\begin{array}{l}\text { Mean Slop } \\
\text { Median Sl } \\
\text { SD Slope } \\
\text { Mean } I^{2} \\
\text { Mean } r^{2} \\
\text { N }\end{array}$ & & 1 & & 10 & & 2 & & & \\
\hline
\end{tabular}

"The actual angular speed differed for different spatial periods; see Procedure section for exact values. **The data of Groups I and II were combined for this condition. 
motion constancy was met. The mean correlations between perceived distance and perceived speed were high. As shown in the lower portion of the table, they ranged between 0.85 and 0.95 with a mean of 0.90 . The $r^{2}$ values also shown in the table ranged between 0.74 and 0.91 with a mean of 0.83 . Thus, on the average, only $17 \%$ of the variance was unaccounted for by variations in perceived distance.

\section{DISCUSSION}

The results support our hypothesis that for objectreferred motion perception, perceived distance determines perceived speed according to Equation 1. Furthermore, they show that perceived distance and spatial frequency jointly determine perceived speed. As is clear from Figure 2, the magnitude of $\mathrm{k}$ in Equation 1 increases linearly with increasing spatial frequency. Thus it can be said that spatial frequency potentiates the effect of perceived distance on perceived speed.

The degree to which perceived distance alone, in the absence of a contribution from spatial frequency, affects perceived speed can be inferred from Figure 2. Zero spatial frequency on the abscissa of this figure can be regarded as representing the case in which a single edge moves across the visual field. It can be seen that the function relating $k$ and spatial frequency does not intercept the ordinate at zero. This should be the case, since a single moving edge does possess a definite perceived speed. The fact that the function intercepts the ordinate at $\mathrm{k}=0.58$ can therefore be interpreted as meaning that about $58 \%$ of perfect motion constancy may result when a single edge moving at a constant angular speed varies in perceived distance from the observer. The value $k$ then is composed of two terms: one dependent upon spatial frequency represented by the slope of the function in Figure 2, and the other independent of spatial frequency represented by the $\mathrm{Y}$ intercept. The regression line for this function was calculated to be:

$$
k=\left(5.22 \cdot f_{s}+0.58\right)
$$

where $f_{s}=$ the spatial frequency of the moving pattern. If Equation 3 is substituted into Equation 1, the resulting equation reflects the joint contributions of perceived distance and spatial frequency in the determination of perceived speed:

$$
M^{\prime}=\left(5.22 f_{s}+0.58\right) \omega \dot{D}^{\prime} .
$$

It can be seen from Equation 4 that in the presence of a single moving edge, the frequency term drops out, since $f_{s}=0$. In this case, perceived speed would be determined only by $\omega$ and $\mathrm{D}^{\prime}$ according to the relationship $\mathrm{M}^{\prime}=0.58 \omega \mathrm{D}^{\prime}$. It is also clear from Equation 4 that variations in spatial frequency alone lead to variations in perceived speed. This conse-

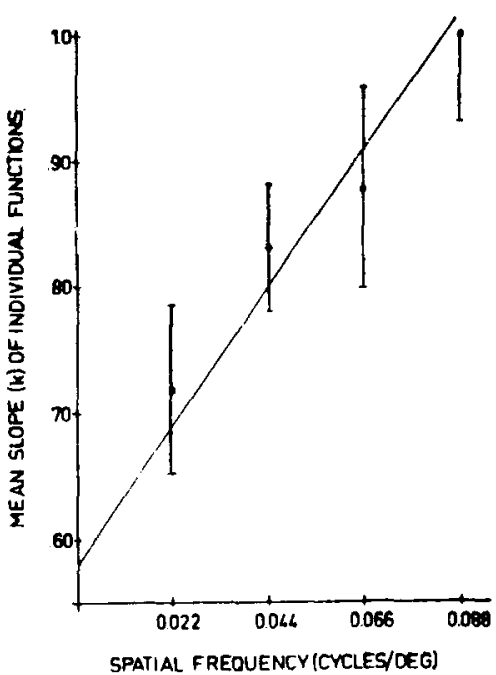

Figure 2. The relationship between the spatial frequency $\left(f_{;}\right.$of the moving stimulus pattern and the mean slope, $k$, of Equation 1 $\left(M^{\prime}=k \omega D^{\prime}\right)$. Slopes were calculated from the individual data and then averaged. Vertical lines represent standard deviations. The diagonal line was fitted to the points by the method of least squares.

quence has already been verified in a previous study in which $D^{\prime}$ was held constant while $\omega$ and $f_{5}$ were independently varied (Diener et al., 1976). It was found that a doubling of $f_{s}$ resulted in an increase in $M^{\prime}$ which was $61 \%$ of that which should have been obtained if a doubling of $f_{s}$ had lead to a doubling of $M^{\prime}$, i.e., if a perfect relation between $f_{S}$ and $M^{\prime}$ had existed. The corresponding percentage value for the present study was $68 \%$. Taking into account the differences in stimulus conditions and methodology between the two studies, these two percentages are quite comparable.

The present results cannot be easily accounted for in terms of the transposition principle. The dimensions of the stimulus field and of the moving stripes were not physically altered by the presence of the neutral density filter. Through the filter, the stripes appeared nearer and narrower, while the stationary borders of the stimulus field were unaffected. Given these conditions, on the basis of the transposition principle, the stripes ought to have appeared to be moving faster (Brown, 1931). Since, in fact, they appeared to be moving more slowly, some other mechanism must have been in operation. An alternative hypothesis might be that the reduced perceived width of the stripes at nearer perceived distances results in reduced perceived speed via a size constancy mechanism. This account, however, is unlikely, since the results of Diener et al. (1976) indicate that it is not the perceived size (or perceived spatial frequency) of a pattern which determines its perceived speed, but rather the temporal frequency of stimulation, which remains unaltered when perceived distance is varied by means of the Pulfrich 
stereoeffect. Furthermore, we have found that the spatial frequency effect disappears during pursuit eye movements (Dichgans, Wist, Diener, \& Brandt, 1975). It is highly improbable that size constancy also disappears during ocular pursuit of the moving pattern.

The present results are consistent with the interpretation that for a given spatial frequency the perceived distance of the stripes per se determines perceived speed in accordance with Equation 4. Moreover, a similar effect of perceived distance on perceived speed has been demonstrated in the case of self-referred motion (circularvection) (Wist, Diener, Dichgans, \& Brandt, 1975). Additional evidence strongly supporting this interpretation was obtained in a supplementary experiment, in which it was found that, in the presence of a single moving vertical edge traversing most of the visual field at a constant angular speed, perceived speed increases with increasing perceived distance. A perceived size interpretation is not applicable to the single-edge case.

The spatial frequency effect on perceived speed was shown in our previous study to be consistent with the interpretation that it is mediated by temporal frequency and that constant temporal frequency could be used by the visual system to signal constant linear speed (Diener et al., 1976). While neither spatial frequency nor perceived distance alone is adequate to produce perfect motion constancy, acting together, perfect motion constancy may be achieved. Paradoxically, Figure 2 implies that, with further increases in the spatial frequency of the moving pattern, overconstancy will result, assuming that this function remains linear. Whether this is, in fact, the case remains to be determined. Under normal environmental conditions, however, overconstancy for perceived speed is unlikely, both because of the falloff of perceived distance with increasing physical distance (Gilinsky, 1951) and because of the visual system's inability to resolve moving patterns of high spatial frequencies (Watanabe, Mori, Nagata, \& Hiwatashi, 1968).

Finally, it must be stressed that, on the basis of the present data, the generality of the spatial frequency contribution to motion constancy is limited. It applies only to the case where fixation is maintained on a stable point in the environment, i.e., to afferent motion perception. We have found that when the eyes pursue the moving pattern (efferent motion perception) the spatial frequency effect on perceived speed disappears (Dichgans et al., 1975). It is plausible, however, under normal environmental conditions when the eyes pursue a moving target, that the spatial frequency of the stationary background (or foreground) may affect the perceived speed of the tracked object. In this case, the background sweeps across the retina in the direction opposite to that of the pursuit movements of the eyes, thus producing an afferent motion stimulus. If this afferent motion information were to combine with the efferent information from the motor pursuit commands (Efferenzkopie, von Holst \& Mittelstaedt, 1950; corollary discharge, Sperry, 1950; Teuber, 1960), a spatial frequency effect on perceived speed for efferent motion perception would result. While it is true that the spatial frequency of the background pattern is a function of the depth separation between it and the tracked object, its temporal frequency would be independent of this separation. This is true because an increase in the absolute distance of the background from the retina would result in a decrease in its angular speed which would exactly compensate for its increase in spatial frequency. The possible existence of such an interaction between efferent and afferent motion information in the determination of perceived speed was recently investigated (Dichgans et al., 1975). No influence of the temporal frequency of the background pattern on efferent motion perception was found. Apparently, the temporal frequency effect on perceived speed exists only for afferent motion perception. During smooth pursuit eye movements, afferent information from the background is not combined with efferent motion information.

\section{REFERENCE NOTE}

1. Wist. E. R., Brandt, Th.. Dichgans, J., and Diener. H.-C. The Pulfich stereophenomenon is dependent upon the spatial period of the moving stimulus pattern. Paper presented at the meeting of the Psychonomic Society. Denver. November 1975.

\section{REFERENCES}

Brown, J. F. The visual perception of velocity. Psychologische Forschung, 1931, 14, 199-232.

Dichgans, J., Wist, E. R., Diener, H.-C., \& Brandt, Th. The Aubert-Fleischl phenomenon: A temporal frequency effect on perceived velocity in afferent motion perception. Experimental Brain Research, 1975, 23, 529-534.

Diener, H.-C., Wist, E. R., Dichgans, J. \& Brandt, Th. The spatial frequency effect on perceived velocity. Vision Reseanch, 1976, 16, 169-176.

Grl.nski, A. S. Perceived size and distance in visual space. Psychological Review, 1951, 58, 460-482.

Kilpatrick, F. P., \& Itrelson, W. H. The size-distance invariance hypothesis. Psvchological Review, 1953, 60, 223-230.

LrT, A. The magnitude of the Pulfrich stereophenomenon as a function of binocular differences of rentinal illuminance at scotopic and photopic levels. American Joumal of Psychology, $1949,62,159-181$.

Pulfrich, C. Die Stereoskopie im Dienst der isochromen und heterochromen Photometrie. Naturwissenschafien, 1922 , 10. $553-564$.

Rock. I.. Hill, A. L., \& Fineman, M. Speed constancy as a function of size constancy. Perception \& Psychophysics, $1968,4,37.40$.

SPerry, R. W. Neural basis of the spontaneous optokinetic response produced by visual inversion. Joumal of Comparative and Physiological Psychology. 1950, 43, $482-489$. 
Stevens, S $S$ On the psychophystcal law. Psychological Revie'w, 1957, 64, 15.3.181.

Teuner, H. L. Perception. In J. Field (Ed.), Handbook of physiology: Neurophysiology (Vol. III). Baltimore: Williams and Wilkins, 1900

von Holst, E., \& Mittelstaedt, H Das Reafferenzprinzip (Wechselwirkung zwischen Zentralnervensystem und Peripherie). Natumissenschatien, 1950, 37, 464-476.

Wallach, H. On constancy of visual speed. Psychological Review, 1939, 46, 547552.

Watanabe, A., Mori, T., Nagata, S., \& Hiwatashi, $K$. Spatial sine-wave responses of the human visual system. Vision Research, 1968, 8, 1245-1263.

Wist, E. R.. Diener, H.-C.. Dichgans, H., \& Brandt, Th. Perceived distance and the perceived speed of self-motion: Linear versus angular velocity? Perception \& Psychophysics. $1975,17,549.554$.

\section{NOTES}

1. Actually, two conditions must be met before perfect motion constancy can be said to exist. Not only must (a) $\mathrm{k}=1$, in Equation 1 , but also (b) perceived speed must correspond exactly to physical (linear) speed. Thus, while (a) is a necessary condition for perfect motion constancy, it is not a sufficient condition. Only (a), which is reflected in Equation 1, was assessed in the present study.

2. The fact that the angular speed required to produce a given shift in the depth plane of the moving pattern via the Pulfrich effect was a function of the pattern's spatial frequency is an interesting phenomenon in its own right. The greater the spatial frequency of the moving pattern, the less the angular speed required to produce a given shift in depth plane. We have investigated this phenomenon more extensively in a subsequent study, which will be published separately (Wist, Brandt, Dichgans, \& Diener, Note 1).
3. An effect of a luminance increase could be observed with monocular viewing if the $1.5 \mathrm{log}$ unit filter were held in place longer than the $15-\mathrm{sec}$ period used here before removal. This effect will be dealt with in a subsequent paper. Apparently, sufficient time for adaptation to the lower luminance must be allowed before an apparent speed increase is observed upon removal of the filter. This apparent speed increment, however, is much smaller than that obtained after binocular viewing with the filter covering one eye. Even if some small effect of luminance had existed in the present study, it could not have been responsible for the results obtained. This is true because the luminance change coincident with the introduction or removal of the $1.5 \mathrm{log}$ unit filter was constant for all viewing conditions, while the apparent speed changes were not. They were correlated instead with the magnuudes of perceived distance changes.

4. Preliminary experiments, conducted with three subjects, in which the modulus for both speed and distance estimates was " 100 " when the standard stimulus was located perceptually at the distance of the screen yielded results similar to those reported here.

5 . The reason that the influence of spatial frequency on perceived speed is more clearly seen in Figure 2 than in Figure 1 hinges on the fact that a sizable intersubject variation in perceived distance produced by the Pulfrich effect for a given angular speed existed. This meant that even if the individual functions for two subjects were identical, different perceived distances (and correspondingly different perceived speeds) were obtained for the same stimulus condition, because different points along these identical functions were being sampled. When the slopes of the individual functions are averaged, however, this source of intersubject variability is eliminated.

(Received for publication May 30, 1975; revision accepted February 17, 1976.) 